\title{
Neutron Production Evaluation from a ADS Target Utilizing the MCNPX 2.6.0 code
}

\author{
Graiciany P. Barros, Claubia Pereira, Maria A.F. Veloso, and Antonella L. Costa \\ Departamento de Engenharia Nuclear - UFMG, \\ Belo Horizonte, MG, Brazil \\ Instituto Nacional de Ciências e Tecnologia de Reatores Nucleares Inovadores/ CNPq \\ Rede Nacional de Fusãol FINEP \\ Patrícia A.L. Reis \\ Departamento de Engenharia Nuclear- UFMG, \\ Belo Horizonte, MG, Brazil \\ Instituto Nacional de Ciências e Tecnologia de Reatores Nucleares Inovadores/ CNPq \\ Rede Nacional de Fusãol FINEP
}

(Received on 24 June, 2010)

\begin{abstract}
Accelerators Driven Systems (ADS) are an innovative type of nuclear system, which is useful for long-lived fission product transmutation and fuel regeneration. The ADS consist of a coupling of a sub-critical nuclear core reactor and a proton beam produced by particle accelerator. These particles are injected into a target for the neutrons production by spallation reactions. This target is of utmost importance for an ADS, representing the coupling of the accelerator and the sub-critical core. The determination of optimal materials for these targets is fundamental for the design of an ADS. The main characteristic of an ideal target is the high production of neutrons per incident proton. In this work are shown results for the neutron production of various types of targets using the MCNPX 2.6.0 code. Furthermore, it is performed a comparative study of transport models to describe the spallation reactions available in this code.
\end{abstract}

Keywords: Spallation, Monte Carlo, transpot models, intranuclear cascade.

\section{INTRODUCTION}

The Accelerator Driven System (ADS) is an innovative nuclear reactor which has been studied to produce energy and transmute radioactive wastes, or as a starter to breed the required ${ }^{233} \mathrm{U}$ in a thorium based type of fuel. A great number of works on the ADSs and on their neutronics has been reported in the scientific literature [1-10]. The concept of ADS combines a particle accelerator with a sub-critical core. The sub-criticality enables that a fuel with a large fraction of fuel and/or minor actinides can be loaded into the subcritical system without any reactivity induced safety problems, which would not be the case in a critical system and that the system can be controlled safely and easily because the sub-critical systems need an external source of neutrons to start the nuclear reactions. An ADS consists of three parts: (1) particle accelerator, (2) spallation target and (3) sub-critical reactor core. A high intensity continuous wave proton beam with an energy of around $1 \mathrm{GeV}$ and a current of several tens milliamperes is injected into a target of heavy metal. This results in a spallation reaction that emits neutrons.

The spallation process is a nuclear reaction where highenergy particles hit target nuclei of heavy elements. The main purpose of the spallation target in an ADS is to provide the primary neutron flux for driving the fission process in the surrounding subcritical core. The number of spallation neutrons per incident proton depends on the beam energy and on the mass of the target nuclei. Due to their high atomic number, heavy metals such as lead, uranium, tungsten or eutectics such as lead-bismuth are the most appropriate choices for the target material. Lead-bismuth eutectic is today the reference target material for ADS applications. Both lead and leadbismuth exhibit very low neutron capture making them good candidates from a neutronic standpoint.
The most widely proposed systems use, for the induction of spallation reactions, high energy protons. A few other propositions resorted to electrons or deuterons as originators of neutron producing reactions. Due to its importance, high energy protons are used in this study.

In 2008, Brasil government created the Instituto Nacional de Cincias e Tecnologia de Reatores Nucleares Inovadores/ $C N P q$ and the Rede Nacional de Fuso/ FINEP with the main aim to study new-generation reactor designs and systems. Among these reactors are the Accelerators Driven Systems considered in this work. In the Departamento de Engenharia Nuclear/ UFMG has also been developed works involving fusion systems [11].

The objective of the present study is to investigate the neutron production in various types of spallation target and to compare several transport models available in the MCNPX 2.6.0 code for the spallation target simulations. The neutron production in a spallation target is an important parameter in an ADS, since the target acts as an external source to the sub-critical system. Therefore, it is essential to quantify the neutron production in the target, since this production will directly influence the ADS criticality. This type of comparative study of several models of particles transport on ADS targets is different from other studies by using the latest version of the MCNPX code. Such version is quite interesting for the simulation of ADS because it allows the description of external sources from spallation reactions and also to monitor the nuclear fuel evolution. In this paper we present the results for neutron production in different types of ADS targets. We further perform a comparative study of the neutron production in a $\mathrm{Pb}-\mathrm{Bi}$ target for various transport models available in MCNPX 2.6.0 code. 


\section{INTERACTION OF PROTONS WITH MATTER}

Looking at a general description of the interaction of protons with a matter $[12,13]$, it can be seen that energetic protons and nuclei interact with matter mostly by collisions with electrons. These lead to progressive energy loss.

The energy loss due to eletronic collisions is given by Bethe's formula which reads, to a very approximation:

$$
\frac{d E}{d x}=\frac{D Z \rho}{A}\left(\frac{Z_{p}}{\beta}\right)^{2}\left(\ln \left(\frac{2 m_{e} c^{2} \gamma^{2} \beta^{2}}{I(Z)}\right)-\beta^{2}\right) M e V / c m
$$

with $A, Z$ and $\rho$ respectively the mass number, the charge and the mass density of the target nucleus and $\mathrm{Zp}, \beta, \mathrm{E}$ respectively the charge, velocity and energy of the projectile. The constant $D=0.3071 \mathrm{MeV} \mathrm{cm}^{2} / \mathrm{g} ; \mathrm{I}$ is the average ionization potential of target atoms, with approximate value $I(Z)=16 Z^{0.9} \mathrm{eV}$. Also $m_{e} c^{2}=0.511 \mathrm{MeV}$.

Bethe's formula does not allow to obtain an analytic expression of the projectile range. A common approximation, which allows reasonable proton range estimates, is

$$
\frac{d E}{d x}=496 \rho \frac{Z}{A} \frac{Z_{p} A_{p}^{0.75}}{E^{0.75}}=c_{R} E^{-0.75} \mathrm{meV} / \mathrm{cm}
$$

where

$$
c_{R}=496 \rho \frac{Z}{A} Z_{p} A_{p}^{0.75} .
$$

The range is obtained by integration of

$$
\frac{d E}{d x}=\frac{E^{0.75}}{c_{R}}
$$

which gives:

$$
R_{e l}(E)=\frac{E^{1.75}}{c_{R}}
$$

Note that the stopping power per atom is proportional to $Z$, and independent of $A$ and $\rho$. For a $1 \mathrm{GeV}$ proton one gets an electronic range is

$$
R_{e l}(E=1 G e V)=205 \frac{A}{Z \rho} .
$$

Taking the examples of beryllium and lead we get, for a 1 $\mathrm{GeV}$ proton,

- for beryllium: $R_{e l}(E=1 \mathrm{GeV})=250 \mathrm{~cm}$

- for lead: $R_{e l}(E=1 G e V)=45 \mathrm{~cm}$

While being slowed down, protons may undergo nuclear reactions. For proton energies larger than, typically, $100 \mathrm{MeV}$, the most violent reactions are called spallation. These account for most of the neutrons produced.

In a crude, black nucleus model, the reaction cross-section reads:

$$
\sigma(E)=\sigma_{0}\left(1-\frac{V_{c}}{E}\right)
$$

with the geometrical cross-section:

$$
\sigma_{0}=\frac{\pi}{100}\left(1.3 A^{\frac{1}{3}}+1\right)^{2}
$$

$V_{c}$ is the coulomb barrier:

$$
V_{c}=\frac{1.44 Z}{1.3 A^{1 / 3}+1} \frac{A+A \rho}{A} .
$$

With these expressions it is possible to derive a nuclear range. For high energy protons the cross-section reduces to the black body value, and, thus, the nuclear range reads approximately: $V_{c}$ is the coulomb barrier:

$$
R_{n u c}=\frac{A}{0.6 \rho \sigma_{0}}+\frac{31 A^{1 / 3}}{\rho} .
$$

Thus, for

$$
\begin{aligned}
& \text { - beryllium } R_{n u c}=35 \mathrm{~cm} \\
& \text { - Lead } R_{n u c}=16 \mathrm{~cm}
\end{aligned}
$$

The probability that $1 \mathrm{GeV}$ protons suffer nuclear reactions is very high both for Beryllium and for Lead. The nuclear range is smaller, relative to the electronic range, for light nuclei. On the other hand, the energy deposited in the target nucleus following a nuclear encounter is larger for heavy targets. The energy deposited in the target nucleus, is proportional to the target thickness, i.e. to $A^{1 / 3}$. It follows that the ratio of nuclear energy loss to electronic energy loss scales like $(A / Z) E^{0.75}$.

The over-simplistic considerations we have just made only intended to give a feeling of the physics of the interaction of high energy protons with nuclei. It showed that the proton energy should be chosen high enough that nuclear energy losses exceed electronic energy losses. More detailed treatment requires the use of nuclear cascade simulations.

This deterministic analysis predicts that the best results for the neutron production will be obtained with target materials of high atomic number. What is further corroborated by the simulations probabilistic using the Monte Carlo method.

\section{METHODOLOGY}

\subsection{ADS Target Simulations in the MCNPX 2.6.0 Code}

The MCNPX code [14] as well as most existing codes used for high energy proton-nucleus reactions are based on the intranuclear cascade (INC) [15]/ Pre-equilibrium [16, 17]/ Evaporation [18]. The philosophies of the INC and EVAP models are very different: The INC calculations follow the history of individual nucleons in a classical or semiclassical manner, while the EVAP calculations follow the deexcitation of the whole nucleus while it decays from one nuclear level to a lower one. The connection between the two approaches is one of the delicate points of high (or intermediate) energy simulations of proton-nucleus reactions. In principle the single particle approach of INC should be justified as long as the wavelength of the incident nucleon is smaller than the nucleon radius, i.e. $\sigma<\frac{\pi r}{2}$ Fermi and $\mathrm{E}_{i} 160 \mathrm{MeV}$. 
On the other hand, the evaporation approach is valid as long as the energy of the nucleon does not exceed too much the nuclear potential depth, i.e. about $40 \mathrm{MeV}$. Thus, the transition energy between the INC and EVAP calculations cannot be specified rigorously. For that matter several codes, as well as MCNPX, have added an intermediate step whose domain of validity is expected to overlap on the INC and EVAP domains. This step is the preequilibrium step (PE).

In the INC the incident proton collides with one or several nucleons of the target nucleus. The struck nucleons, in turn, collide with the unperturbed nucleons. A cascade develops. The INC calculation for a specific nucleon stops whenever its energy falls below a specified value, related to the depth of the nuclear potential well.

The INC model lacks justification for nucleon energies (inside the nucleus) below around 100-150 MeV. Preequilibrium models have been used in nuclear physics in this energy domain. These models follow a population of quasiparticle excitations of the nuclear Fermi gas by means of a master equation. Quasi-particle states are characterized by their particle escape and damping widths. Angular distributions are associated to the escaping particles. In a sense, preequilibrium models allow an easier phenomenological adjustment of angular distributions than does the intranuclear cascade.

\subsection{The Target Design and Beam Profile}

One of the fundamental quantities of interest in most spallation target applications is the number of neutrons produced per beam particle incident on target. For targets fed by proton accelerators, this quantity is typically denoted as "n/p". In this work this calculation of the neutron production is made for several types of target materials using MCNPX. The geometry consists of a simple right circular cylinder, $30 \mathrm{~cm}$ in diameter by $50 \mathrm{~cm}$ long. A beam of $1-\mathrm{GeV}$ protons is launched onto the target. The beam has a 7-cm-diameter spot size, with a parabolic spatial profile. (See Figure 1)

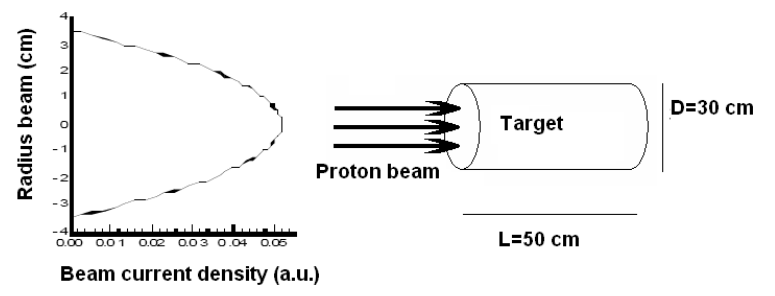

FIG. 1: Schematic representation of the target and beam.

In MCNPX, net neutron production is tallied implicitly and is provided by default in the problem summary for neutrons [14]. The problem summary shows net neutron production resulting from nuclear interactions (the component that accounts for neutron production by all particles transported using INC/Preequilibrium/Evaporation physics) and net production by $(\mathrm{n}, \mathrm{xn})$ reactions (neutrons created in inelastic nuclear interactions by neutrons below the transition energy, using evaluated nuclear data). Net production from nuclear interactions is given by the difference of the neutron weights in the "neutron creation" and "neutron loss" columns. A similar approach is taken to calculate net $(\mathrm{n}, \mathrm{xn})$ production. Net neutron production may also be calculated by realizing that the only loss mechanisms for neutrons are escape and capture. The sum of the weights in the "neutron loss" column under "escape" and "capture" is thus equal to the net neutron production. The values listed in the problem summary are "collision estimators", meaning they are tallied when a collision occurs during transport. Uncertainties are not calculated by MCNPX for these collision-estimated quantities. A reasonable upper limit on the relative uncertainty would be given by the inverse square root of the number of source particles launched. In our simulations the number of source particles launched is 20,000. Therefore, a reasonable upper limit on the relative uncertainty of $\mathrm{n} / \mathrm{p}$ is $20,000^{-1 / 2} \approx 0.7 \%$.

\section{RESULTS AND ANALYSIS}

\subsection{Neutrons Production for various target materials}

For comparison purpose were modeled several types of target materials in natural compositions. The parameters of beam and cylinders for all simulations were them described in section IV. In these simulations were transported protons, neutrons, and charged pions. The ENDF/B-VI.6 neutron libraries are used. All protons are transported using LAHET physics. Nucleon and pion interactions simulated by LAHET physics use the Bertini intranuclear cascade model. The results for neutron yield from different target materials and an upper limit on the relative uncertainty are showed in the Table 1 .

The analysis of this table shows that the neutron production is larger for targets of high atomic number. This behavior was already expected, once the nuclear range is smaller, relative to the electronic range, for light nuclei.

TABLE I: Neutron multiplicity for $1 \mathrm{GeV}$ protons.

\begin{tabular}{lcc}
\hline Material & $(\mathrm{n} / \mathrm{p})$ & Uncertainty \\
\hline $\mathrm{Pb}-82$ & 26.23 & 0.18 \\
$\mathrm{U}-92$ & 58.13 & 0.41 \\
$\mathrm{Th}-90$ & 36.46 & 0.25 \\
$\mathrm{Bi}-83$ & 24.75 & 0.17 \\
$\mathrm{Li}-3$ & 0.98 & 0.01 \\
$\mathrm{Be}-4$ & 4.03 & 0.03 \\
$\mathrm{~W}-74$ & 29.48 & 0.21 \\
$\mathrm{~Pb}-\mathrm{Bi}$ & 24.86 & 0.17 \\
\hline
\end{tabular}

Among these targets the more commonly used are the lead and the eutectic mixture of lead-bismuth, since the neutron production is larger when liquid target are used, and the melting points of these materials are relatively low. The use of liquid uranium would be unviable due to its high melting point $(1405.3 \mathrm{~K})$. Lead-bismuth eutectic (LBE) is preferred as target material due to its high production rate of neutrons, effective heat removal and a very small amount of radiation damage properties. The melting temperature of LBE (396.5 $\mathrm{K})$ is smaller than when just used lead $(600 \mathrm{~K})$. Bismuth 
leads to ample production of the very radiotoxic and volatile ${ }^{210} \mathrm{Po}$. It is also produced, but at a very much lower rate, by lead. However, since it is possible for the lead bismuth target to work at much lower temperature than pure lead the evaporation rates of ${ }^{210}$ Po can be similar in both cases [19].

Due to the relevance of LBE it was made a more detailed study of this target. The cost of the proton accelerator is an important parameter when designing a neutron spallation source to have a comparative, or to evaluate a merit figure, an interesting parameter is the "neutron value", which is defined as the number of produced neutrons normalized to the unit beam energy per incident particle. Figure 2 illustrate such parameter. For these simulations it was also used the Bertini intranuclear cascade model and were transported protons, neutrons, and charged pions.

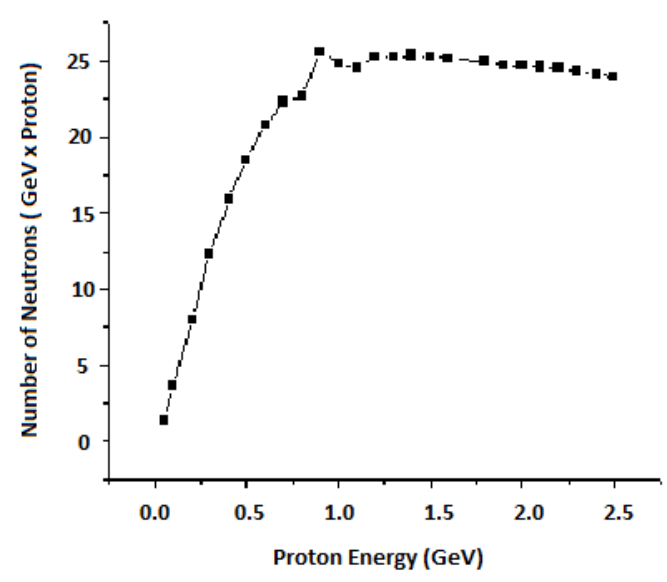

FIG. 2: Neutron multiplicity per unit energy and per incident proton as a beam energy for a LBE target.

From these results we notice that a maximum occurs in the range of energy of $0.75-1.25 \mathrm{GeV}$, therefore this is the range of protons energy that provides a maximum "neutron value", defining the optimum conditions, when economy is considered.

\subsection{A Comparative study of the transport models available in MCNPX 2.6.0 code for $L B E$ target}

MCNPX offers several other transport models for the modeling of spallation reactions. For a comparative study were made simulations of these models for a $\mathrm{PbBi}$ target following the parameters of the cylinder and beam described in section IV. For the "base case" described before it was used Bertini intranuclear cascade model for the transport of protons, neutrons, and charged pions. For this case the neutron production was $26.23 \mathrm{n} / \mathrm{p}$.

In a first variation it was used the model Bertini, as done previously, but with the transport of light ions (deuterons, tritons, ${ }^{3} \mathrm{He}$, and alphas). Net neutron production for this case was $24.92 \mathrm{n} / \mathrm{p}$, or $0.24 \%$ above the case 1 value. That is, the inclusion of the light ions transport did not change much the neutron production. The implication of this result is that we need not concern ourselves with light ion transport if the quantity with which we are concerned is related solely to neutrons, as neutron production by light ions is small when we start with a proton beam.

In the second variation, we replaced the Bertini INC model used in the base case for the simulation of nucleon and pion interactions with nuclei by the ISABEL INC model. The net neutron production calculated with the ISABEL INC model is $23.60 \mathrm{n} / \mathrm{p}$, which is $5.06 \%$ below the value predicted by the Bertini INC model. This is consistent with other studies that reveal slightly lower neutron production resulting from ISABEL as compared to Bertini [14].

In the third variation, we use the CEM model for neutron, protons and pions. Note that CEM, unlike the other INC models in the code, has its own evaporation model (GEM). Therefore, the pre-equilibrium (MPM) model and evaporation model settings have no effect when CEM is specified. Note the net neutron production calculated with the CEM model was $25.78 \mathrm{n} / \mathrm{p}$, which is $3.7 \%$ above the value predicted by the Bertini INC model.

In the final variation from the case 1 we used the INCL model coupled with the ABLA evaporation mode. The ABLA evaporation model is automatically chosen when INCL is specified. Net neutron production for this case was 24.29 n/p, $2.29 \%$ less than the case 1 value.

Results compiled for each case of this example are shown in Table 2. Runtimes were obtained using MCNPX 2.6.0 on a $2.33 \mathrm{GHz}$ PC running Open SUSE Linux distribution.

TABLE II: Results Compiled for Summary Cases for a LBE Target.

\begin{tabular}{|c|c|c|c|c|}
\hline Case & INC Model & Particles Transported & Runtime (minutes) & $(\mathrm{n} / \mathrm{p})$ \\
\hline Base & Bertini & N H / & 4.91 & 26.23 \\
1 & Bertini & N H / D T S A & 5.61 & 24.92 \\
2 & ISABEL & N H / & 5.87 & 23.60 \\
3 & CEM & N H / & 5.19 & 25.78 \\
4 & INCL & N H / & 50.92 & 24.29 \\
\hline
\end{tabular}

Notice the runtime for the case where the ISABEL INC model is used is about $15 \%$ greater than the base case using the Bertini model. Case 2 also runs slower because more particles are tracked and the light ion interactions are provided by the ISABEL model. The INCL model has a significant speed penalty, almost a factor of 10 .

These results demonstrate the different models options for ADS targets simulation using the MCNPX code and the different results obtained for the neutron production for each of these models. When the quantity of interest depends only on neutrons and one starts with a proton beam, there is no need to transport any particles other than protons, neutrons, and charged pions, as neutron production by other particles is negligible compared to production by these three particle types.

\section{CONCLUSION}

The results show that the neutron production is larger for targets of high atomic number. The results also show that the 
higher neutron production in a LBE target occurs when using protons with range of energy of $0.75-1.25 \mathrm{GeV}$.

The comparative study for LBE target shows that the use of the various physics model options for the modeling of spallation reactions, such as the ISABEL, CEM, INCL mod- ules, within MCNPX allows the user to test the sensitivity of the quantity of interest to the different physics models. If significant differences are observed, the user should evaluate which physics model is most appropriate for the particular application.
[1] C. D. Bowman et al., Nuclear energy generation and waste transmutation using an accelerator-driven intense thermal neutron source, Nucl. Instr. And Methods A, 320, (1992), 336367.

[2] F. Carminati et al.,An energy amplifier for cleaner and inexhaustible nuclear energy production driven by a particle beam accelerator, CERN/AT/93-47 (ET), 1992.

[3] C. Rubbia et al., Conceptual design of a fast neutron operated high power energy amplifier, CERN/AT/95-44 (ET), 1995.

[4] C. Bowman, Accelerator-Driven Systems for Nuclear Waste Transmutation, Annu. Rev. Nucl. Part. Sci., 48, (1998), 505556.

[5] S. David et al., Fast subcritical hybrid reactors for energy production: evolution of physical parameters and induced radiotoxicities, Nucl. Instr. And Methods A, 443, (2000), 510530.

[6] K. tsujimoto et al., Neutronics design for lead-bismuth cooled accelerator-driven system for transmutation of minor actinide, Journal of Nuclear Science and Technology, 41, (2004), 2136.

[7] D. Cacuci, On the Neutron Kinetics and Control of Accelerator-Driven Systems, Nuclear Science and Engineering, 148, (2004), 55-66.

[8] K.Tsujimoto et al., Feasibility Of Lead-Bismuth Cooled Accelerator-Driven System For Minor-Actinide Transmuta- tion, Nuclear tecnology 161,(2007).

[9] B. Calgaro et al., Wastes Management Through Transmutation in an ADS Reactor, Science and Technology of Nuclear Installations, (2008).

[10] H. Yapici et al.,A comprehensive study on neutronics of a lead-bismuth eutectic cooled accelerator-driven sub-critical system for long-lived fission product transmutation, Annals of Nuclear Energy 35, (2008),1264-1273.

[11] A. Arajo et al., Flux and Dose Rate Evaluation of ITER System Using MCNP5, Brazilian Journal of Physics 40, (2010), 683-827.

[12] H. Nifenecker et al., Hybrid Nuclear Reactors, Progress in Particle and Nuclear Physics 43, (1999), 50-62.

[13] H. Nifenecker et al., Accelerator Driven Subcritical Reactors (Betts-Greiner) (2003).

[14] D. Pelowitz, Editor, MCNPX users manual, LA-CP-07-1473 Version 2.6.0, Los Alamos National Laboratory, 2008.

[15] H. Bertini, Phys. Rev., 188, (1969), 1711.

[16] M. Blann, Ann. Rev. Nucl. Sci., 25, (1975), 123.

[17] H. Feshbach et al., Ann. Phys., 125, (1980), 429.

[18] I. Dostrovski et al., Phys. Rev., 116, (1959), 683.

[19] H. Nifenecker et al., Basics of accelerator driven subcritical reactors, Nucl. Instr. And Methods A, 463, (2001), 428-467. 